\title{
Up-Regulation of the miR-92a and miR-181a in Patients with Acute Myeloid Leukemia and their Inhibition with Locked Nucleic acid (LNA)-anti- miRNA; Introducing c-Kit as a New Target Gene
}

\author{
Mahdiyar IRAVANI SAADI ${ }^{1}$, Nargess ARANDI ${ }^{1}$, Ramin YAGHOBI $^{2}$, Negar AZARPIRA ${ }^{2}$, \\ Bita GERAMIZADEH ${ }^{2}$, Mani RAMZI ${ }^{1}$ \\ ${ }^{1}$ Shiraz University of Medical Sciences, Hematology Research Center \\ ${ }^{2}$ Shiraz University of Medical Sciences, Transplant Research Center, Shiraz, IRAN
}

\begin{abstract}
Dysregulated expression of various microRNAs (miRNAs) has been widely observed in hematopoietic malignancies like acute myeloid leukemia (AML). In this study, we evaluated the expression of the miR-92a and miR-181a in newly diagnosed AML patients compared to healthy controls. Also, we investigated for the first time the effect of blocking of the miR-92a and miR-181a on the expression of cKit, CEBPA and WT1 genes in HL-60 cell line. For evaluation of the relative gene expression, SYBR Green Real-Time PCR method was performed. The expression of miRNAs was inhibited by transfection of the HL-60 cell line with locked nucleic acid (LNA)-anti-miRNA. The viability of transfected cells was evaluated by MTT assay. Both miR-92a and miR-181a are highly overexpressed in AML patients compared to healthy controls. Also, miR-181a expression was associated with poor prognosis of AML patients. The blockage of the miR-92a and miR-181a remarkably reduces cell viability. In addition, inhibition of miR-92a with LNA-anti-miR-92a significantly decreased c-Kit level. Conversely, miR-181a blockage was associated with upregulated c-Kit expression. Taking together, miR-92a and miR-181a are dysregulated in AML patients and c-Kit gene might be a novel target for these miRNAs. Regarding the anti-proliferative effect of LNA-anti-miR-92a and LNA-anti-miR-181a, regulation of miR-92a and miR-181a expression might be a useful approach in line with conventional chemotherapy to limit blast cell survival and reduce leukemic cell proliferation.
\end{abstract}

Keywords: MicroRNA; Acute myeloid leukemia (AML); locked nucleic acid (LNA)-anti-miRNA; Gene expression

\section{INTRODUCTION}

MicroRNAs (miRNAs) are small 18-24 bp dsRNAs that can post-transcriptionally regulate the majority of protein-coding as well as non-coding genes in different cellular processes. ${ }^{1}$ In cancer, miRNAs have been shown to play fundamental roles by regulating different aspects of cancer cell behaviors including cell division and self-renewal, apoptosis, invasion, and DNA damage, thus may act as either oncogene or tumor suppressor gene. ${ }^{2}$ In addition, it has been demonstrated that miRNAs may have a predictive value for response to chemotherapy treatment and disease prognosis in cancers. ${ }^{3}$ Locked nucleic acid (LNA), is a kind of modified RNA nucleotide. Recently, the LNA-antimiRs have been widely used in several studies for inhibition of miRNAs, suggesting them as suitable candidates for therapeutic applications. ${ }^{4}$

Acute myeloid leukemia (AML) is a cancer of the myeloid lineage of blood cells, characterized by the accumulation of abnormal myeloid cells in bone marrow that interfere with normal blood cells development. 
In addition to the genomic change including chromosomal translocation and/or inversion and somatic gene mutations, the role of epigenetic alteration in the pathogenesis of AML has been well documented. ${ }^{5,6}$ Recently, it has been shown that miRNAs have a critical role in controlling the expression of various genes affecting commitment and differentiation of hematopoietic stem cells and aberrant expression of miRNAs has been observed in hematologic malignancies. ${ }^{7-9}$

The miR-181 family (consisted of four conserved miRNAs; miR-181a/b/c/d), are among the miRNAs that are particularly expressed in hematopoietic cells. ${ }^{5}$ It has been demonstrated that the miR-181 family members act as a key regulator of normal cell differentiation, especially those of hematopoietic origin, including $\mathrm{T}$ cells, $\mathrm{B}$ cells, natural killer cells (NK cells) and megakaryocytes. ${ }^{7}$ There are accumulating evidence describing that miR-181 is tightly linked to the pathogenesis and prognosis of AML with significant impact on the survival of AML patients.,

The miR-17/92 cluster (also known as "oncomiR-1"), the first discovered oncogene, is divided into four different families including miR-17 family, miR-18 family, miR-19 family, and miR-92 family. ${ }^{10}$ The miR-92a family is a group of highly conserved miRNAs including miR-25, miR-92a-1, miR-92a-2 and miR-363, which have a critical role in regulating the development of mammalian organs including heart, lungs and immune system as well as the formation of blood vessels. ${ }^{10,11}$ Abnormal expression of miR-92 has been observed in hematological malignancies especially AML patients which may have prognostic value. ${ }^{10,12,13}$
In this study, we evaluated the expression of the miR-181a and miR-92a in peripheral blood mononuclear cells (PBMCs) of the newly diagnosed AML patients compared to healthy controls. Also, we investigated the effect of blocking miR-181a and miR-92a by LNA-anti-miRNA on the expression of some important genes which have dysregulated expression in AML disease including WT1, CEBPA and c-Kit genes in HL-60 cell line.

\section{PATIENTS and METHODS}

\section{Patients' Criteria}

In this cross-sectional study, 100 newly diagnosed adult AML patients were enrolled who referred to our referral Hospital during 2014-2016. The AML disease was diagnosed by an oncologist using morphology, cytochemistry, and immunophenotyping. Clinical and laboratory data including FrenchAmerican-British (FAB) subclass, complete blood count, blast percentage in bone marrow and hemoglobin $(\mathrm{Hb})$ level, were also collected. From 100 AML patients, 38 had normal cytogenetic $(\mathrm{CN}$ AML) and the remaining 62 patients had cytogenetic abnormality (Table 1). Since the FAB group of some AML patients with abnormal cytogenetics was not defined, we divided them into M3 and nonM3 groups. Accordingly, 21 patients were categorized in M3 and 41 in the non-M3 FAB subgroup.

All patients received standard induction chemotherapy, which consisted of daunorubicin $45 \mathrm{mg} /$ $\mathrm{m}^{2}$ on days 1 to 3 and cytarabine $100-200 \mathrm{mg} / \mathrm{m} 2$ on days 1 to 7 , followed by high doses of a cytarabine-based consolidation phase (Cytarabine $3 \mathrm{gm} /$ $\mathrm{m} 2$ every 12 hours for 3 days, repeated for 2 to 3 cycles). For M3 patients, arsenic trioxide $(0.15 \mathrm{mg} /$

\begin{tabular}{|ll|}
\hline Table 1. The cytogenetic status of the AML patients \\
\hline Cytogenetic abnormalities & No. (\%) \\
\hline t(8;21)(q22;q22); RUNX1-RUNX1T1 & $17(17 \%)$ \\
inv(16)(p13.1q22) or t(16;16)(p13.1;q22); CBFB-MYH11 & $14(14 \%)$ \\
t(15;17)(q22;q12); PML-RARA & $21(21 \%)$ \\
t(9;11)(p22;q23); MLLT3-MLL & $10(10 \%)$ \\
Normal cytogenetic (CN-AML) & $38(38 \%)$
\end{tabular}




\begin{tabular}{|c|c|c|}
\hline Primers & Primer sequences (5'->3') & PCR program \\
\hline miR-181a:F & ACTGACAACATTCAACGCTGTCG & $\begin{array}{l}94^{\circ} \mathrm{C} / 2 \mathrm{~min}, 40 \text { cycles of } \\
94^{\circ} \mathrm{C} / 10 \mathrm{sec}, 57.5^{\circ} \mathrm{C} / 20 \mathrm{sec}\end{array}$ \\
\hline miR-181a:R & GTGCAGGGTCCGAGGT & and $72^{\circ} \mathrm{C} / 15 \mathrm{sec}$ \\
\hline miR-92a:F & GTGGTAGGTTGGGATCGGT & $\begin{array}{l}94^{\circ} \mathrm{C} / 2 \mathrm{~min}, 40 \text { cycles } \\
\text { of } 94^{\circ} \mathrm{C} / 30 \mathrm{sec}, 56.5^{\circ} \mathrm{C} / 20 \mathrm{sec}\end{array}$ \\
\hline miR-92a:R & GTGCAGGGTCCGAGGT & and $70^{\circ} \mathrm{C} / 30 \mathrm{sec}$ \\
\hline GAPDH:F & GGACTCATGACCACAGTCCA & $\begin{array}{l}95^{\circ} \mathrm{C} / 2 \mathrm{~min}, 40 \text { cycles } \\
\text { of } 95^{\circ} \mathrm{C} / 30 \mathrm{sec}, 57.5^{\circ} \mathrm{C} / 20 \mathrm{sec}\end{array}$ \\
\hline GAPDH:R & CCAGTAGAGGCAGGGATGAT & and $70^{\circ} \mathrm{C} / 30 \mathrm{sec}$ \\
\hline WT1: F & CCAGGCTITGCTGCTGAG & $\begin{array}{l}95^{\circ} \mathrm{C} / 2 \mathrm{~min}, 40 \text { cycles } \\
\text { of } 95^{\circ} \mathrm{C} / 30 \mathrm{sec}, 57.5^{\circ} \mathrm{C} / 20 \mathrm{sec}\end{array}$ \\
\hline WT1: R & GTGGCTCCTAAGTTCATCTG & and $70^{\circ} \mathrm{C} / 30 \mathrm{sec}$ \\
\hline $\mathrm{C}-\mathrm{KIT}: \mathrm{F}$ & TTCTGCTCCTACTGCTTC & $\begin{array}{l}95^{\circ} \mathrm{C} / 2 \mathrm{~min}, 40 \text { cycles } \\
\text { of } 95^{\circ} \mathrm{C} / 30 \mathrm{sec}, 59.5^{\circ} \mathrm{C} / 20 \mathrm{sec}\end{array}$ \\
\hline C-KIT:R & CTGGATGGATGGATGGTG & and $70^{\circ} \mathrm{C} / 30 \mathrm{sec}$ \\
\hline CEBPA :F & GAAGCACGATCAGTCCAT & $\begin{array}{l}95^{\circ} \mathrm{C} / 2 \mathrm{~min}, 40 \text { cycles } \\
\text { of } 95^{\circ} \mathrm{C} / 20 \mathrm{sec}, 59.5^{\circ} \mathrm{C} / 20 \mathrm{sec}\end{array}$ \\
\hline CEBPA:R & GCCAGATACAAGTGTTGATAT & and $70^{\circ} \mathrm{C} / 30 \mathrm{sec}$ \\
\hline
\end{tabular}

$\mathrm{kg} /$ day IV) was used until bone marrow remission occurs plus ATRA ( $45 \mathrm{mg} / \mathrm{m} \mathrm{2/day)}$ in 2 divided doses in addition to standard induction chemotherapy regiment. Forty age/sex-matched healthy volunteers without a history of malignancies and other diseases were enrolled as healthy control group.

\section{Sample collection and ribonucleic acid isolation}

Five-milliliter peripheral blood was collected in Ethylenediaminetetraacetic acid (EDTA)-containing tubes from each patient at the time of diagnosis prior to chemotherapy treatment as well as control group. The peripheral blood mononuclear cells (PBMCs) were isolated from each individual using Ficoll-hypaque density gradient centrifugation. Total RNA was extracted by Trizol (Invitrogen) and the quantity of the extracted RNA was measured by Nanodrop (ThermoFisher Scientific, USA). Then, total RNA was converted into cDNA using Prime Script RT Reagent Kit (Takara, Japan) according to the manufacturer's instruction in the
T100 thermocycler (Bio-Rad Laboratories, USA) by specific stem-looped designed primers for each miRNAs.

\section{SYBR green real-time PCR}

The relative expression of the miR-92a and miR181a mRNAs was evaluated by the SYBR Green Real-Time PCR method using SYBR® Premix Ex

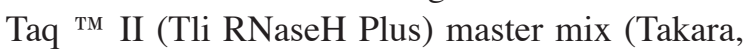
Japan) and designed primers specific for each miRNA in an iQ5 thermocycler (BioRad Laboratories, USA). Glyceraldehyde 3-phosphate dehydrogenase (GAPDH) gene was used as the internal control and the expression of the miR-92a and miR181a mRNAs was normalized to that. Real-Time PCR reaction program and primer sequences are summarized in Table 2. Melt curve analysis was performed to confirm the specificity of reaction at the end of the program. The changes in the relative expression levels of miR-92a and miR-181a mRNAs were calculated by $[2-\Delta \Delta \mathrm{Ct}]$ method, where 
$\Delta \Delta \mathrm{Ct}=[\Delta \mathrm{Ct}$ (patient) $-\Delta \mathrm{Ct}($ control $)]$ and $\Delta \mathrm{Ct}=$ [Ct (sample) - Ct (housekeeping gene)]. All realtime polymerase chain reactions were performed at least in duplicate wells.

\section{Cell culture}

The HL-60 cell line (Human Acute Promyelocytic Leukemia: APL) was purchased from National Cell Bank of Iran (Pasteur Institute, Tehran, Iran). The cells were maintained at Roswell Park Memorial Institute (RPMI) 1640 (Gibco, UK) supplemented with 10-20\% fetal bovine serum (FBS; Gibco), $100 \mathrm{U} / \mathrm{ml}$ of penicillin and $100 \mu \mathrm{g} / \mathrm{ml}$ of streptomycin (Sigma-Aldrich, USA) in an air-saturated and humid atmosphere consisting of $5 \% \mathrm{CO}_{2}$, in $25-\mathrm{cm}^{2}$ culture flasks (Nunc, Denmark), at $37^{\circ} \mathrm{C}$. The cells were passaged twice weekly to maintain an exponential growth phase.

\section{Cell Transfection}

The expression of the miR-92a and miR-181a was inhibited using the LNA perfectly complementary to the $5^{\prime}$ region of miR-92a and miR-181a. The miRNAs nucleotide sequences were obtained from www.mirbase.org (miR-92a: 5'-CAGGCCGGGACAAGUGCAAUA-3' and miR-181a: 5'-ACCCACCGACAGCAATGAATGT-3'). The LNAmiRNA inhibitors for miR-92a, miR-181a and microRNA inhibitor negative control (scrambled) oligonucleotides were purchased from Life Technologies (Applied Biosystems, UK). For cell transfection, the HL-60 cells $\left(2.5 \times 10^{5}\right.$ cells $)$ were cultivated in 6-well plate and after 24 hours reached $80 \%$ confluence. Then, cells were transfected with 50 pmol LNA-anti-miRNA using lipofectamine 2000 reagent (Invitrogen) in serum-free RPMI 1640 medium, according to the manufacturer's instructions. After transfection (about 7 hours), the medium was replaced with fresh complete medium (RPMI with $10 \% \mathrm{FBS}, 100 \mathrm{U} / \mathrm{ml}$ penicillin and $100 \mu \mathrm{g} / \mathrm{ml}$ streptomicin) at $37^{\circ} \mathrm{C}$ in a humidified atmosphere with $5 \% \mathrm{CO}_{2}$. The plates were left up to 48 and 72 hours for real-time quantitative PCR analysis and cell viability analysis, respectively.

\section{Reverse Transcriptase microRNA Real-time PCR}

To determine the successful blockage of the miR92a and miR-181a by LNA-anti-miRs, total RNA of HL-60 cell line transfected with LNA-anti-miR was extracted after $48 \mathrm{~h}$ post transfection using TRIZOL reagent and subsequently converted into cDNA by Prime Script RT Reagent Kit (Takara, Japan). Then, Real-time PCR was performed using SYBR ${ }^{\circledR}$ Premix Ex Taq ${ }^{\text {тм }}$ II (Tli RNaseH Plus) master mix (Takara, Japan) and specific miR-92a and miR-181a designed primers in an iQ5 thermocycler (BioRad Laboratories, USA) and $\Delta \Delta \mathrm{Ct}$ method was used for data calculation.

\section{Measurement of Cell Viability}

To determine the cell viability after transfection, cells will be cultivated after $72 \mathrm{~h}$ post transfection and viability of transfected cells was measured using the MTT assay (3-[4,5-dimethylthiazol2-yl]-2,5-diphenyltetrazolium bromide) (Sigma, Germany). For this purpose, a total of $5 \times 10^{3}$ cells were seeded for 24 hours in 96 well plates and incubated at $37^{\circ} \mathrm{C}$ in a $5 \% \mathrm{CO}_{2}$ incubator. Then, MTT was added and incubation was continued for a further 4 hours at $37^{\circ} \mathrm{C}$. Then, the produced formazan crystals were dissolved in $200 \mu \mathrm{L}$ dimethyl sulfoxide (DMSO) and, afterward, the cells were incubated for further 30 minutes at $37^{\circ} \mathrm{C}$ with gentle shaking. Finally, the absorbance intensity was measured by a microplate reader (FLUOstar Omega, BMG LABTECH, Germany) at $570 \mathrm{~nm}$. Cell viability was calculated as the ratio of the absorbance of the test groups to that of the control group.

\section{Expression of the WT1, CEBPA, and c-Kit}

After confirmation of cell transfection and subsequent successful miR-92a and miR-181a blockage, total RNA of transfected HL-60 cell line with LNA-anti-miRNA and scrambled LNA, as well as non-transfected cells, were extracted and converted into cDNA by Prime Script RT Reagent Kit (Takara, Japan). Then, Real-time PCR was performed using SYBR ${ }^{\circledR}$ Premix Ex Taq ${ }^{\text {тм }}$ II (Tli 


\begin{tabular}{|c|c|}
\hline Variable & Mean \pm SD \\
\hline WBC count & $38572.34 \pm 6856.81$ \\
\hline PLT count & $51849.18 \pm 6626.84$ \\
\hline $\mathrm{Hb}(\mathrm{g} / \mathrm{dl})$ & $8.15 \pm .2$ \\
\hline LDH (U/L) & $1422.65 \pm 242.70$ \\
\hline \multicolumn{2}{|c|}{$\begin{array}{l}S D=\text { standard deviation, } W B C=\text { white blood cells, } P L T= \\
\text { platelet, } H b=\text { hemoglobin, } L D H=\text { lactate dehydrogenase }\end{array}$} \\
\hline
\end{tabular}

RNaseH Plus) master mix (Takara, Japan) and specific WT1, CEBPA and c-Kit designed primers in an iQ5 thermocycler (BioRad Laboratories, USA). GAPDH gene was used as the internal control. Specific primers were designed using Beacon designer software and Primer 3 online softwares. The changes in the relative expression levels of WT1, CEBPA and c-Kit mRNAs were calculated by [2$\Delta \Delta \mathrm{Ct}]$ method.

\section{Statistical Analysis}

Data were analyzed by SPSS software, version 18 . The mean expression of miR-92a and miR-181a were compared between patients and controls by independent $\mathrm{t}$-test. The correlation between miR92a and miR-181a expression and laboratory data was analyzed by Pearson correlation test. The expression of miR-92a, miR-181a, CEBPA, WT-1, and c-Kit were compared between two groups (before and after transfection) by 2-Related-Samples Test. P-values less than 0.05 were considered as statistically significant.

\section{RESULTS}

From 100 newly diagnosed AML patients, 54 (54\%) were male and 46 (46\%) were female. The mean age of AML patients was $38 \pm 2.4$ with a range 20-86 years. Details of demographic and laboratory characteristics of patients are shown in Table
3. Among all AML patients, 33 (33\%) had FLT3ITD mutation.

\section{Expression of the miR-92a and miR-181a in Newly Diagnosed AML Patients}

The mRNA expression of miR-92a and miR-181a was compared between AML patients and controls. After the statistical analysis, our results revealed that the expression of miR-92a was significantly increased (24.2 fold) in AML patients compared to control group $(\mathrm{p}=0.004)$. Also, the expression of miR-181a was significantly higher (34.7 fold) in AML patients compared to group control $(\mathrm{p}<$ 0.001 ). There was no significant correlation between the level of miR-92a and miR-181a expression and response to treatment in our AML patients ( $p>0.05)$.

miR-92a was strongly upregulated in AML patients with cytogenetic abnormalities compared to those with normal karyotype $(\mathrm{p}<0.001)$. There was no significant difference in miR-181a expression between patients with cytogenetic abnormalities and $\mathrm{CN}-\mathrm{AML}$ patients ( $\mathrm{p}>0.05$ ).

Based on the 2017 European Leukemia Net (ELN) genetic risk stratification, AML patients were divided into three groups; favorable, intermediate and high-risk groups. ${ }^{14}$ Accordingly, 32 patients were included in high risk, 46 in intermediate and the remaining 22 in favorable risk groups. The results showed that the mean expression of miR-181a was significantly increased in high-risk group compared to intermediate one $(-4.3 \pm 1.1$ vs. $5.2 \pm 0.57 ; \mathrm{p}=0.001$ ), while no significant difference was observed between high risk and favorable risk as well as favorable risk and intermediate risk group ( $p>0.05)$. The mean expression of miR92a did not significantly change between three risk groups ( $\mathrm{p}>0.05)$.

Regarding FAB subtype, comparison of the miR92a and miR-181a expression between M3 and non-M3 groups demonstrated that the expression of miR-92a and miR-181a did not differ between AML patients with M3 and non-M3 FAB subgroups ( $\mathrm{p}>0.05)$. 


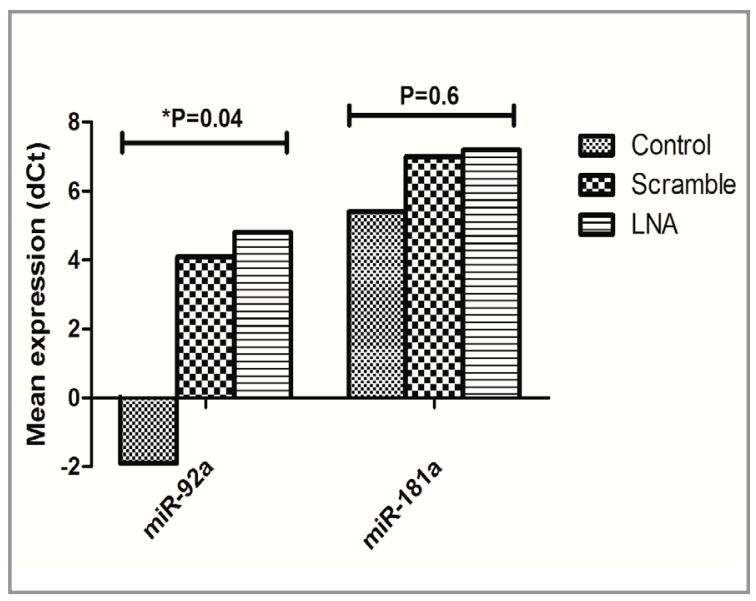

Figure 1. Change in miR-92a and miR-181a expression following HL-60 cell transfection with LNA-anti-miRNA The mean \pm SD of dCt for miR-92a and miR-181a was compared between LNA group and control groups (either scrambles or untreated). Data are represented of the five independent repeated experiments. The P-value is indicative of the comparison between LNA-anti-miR transfected and control group.

\section{LNA-anti-miR-92a and LNA-anti-miR-181a Effectively Inhibit miR-92a and miR-181a Ex- pression}

For determining the efficiency of miRNA blockage by LNA-anti-miRNA, after transfection of HL-60 cell line with LNA-anti-miR92a and LNA-antimiR-181a as well as scrambled LNA (as negative control), the mean expression of miR92a and miR181a was compared between groups transfected with either LNA-anti-miR or scrambled LNA and non-transfected group $48 \mathrm{~h}$ after cell transfection. Although the level of both miRNAs decreased a bit in cells transfected with scrambled LNA group compared with untreated cells, the differences were not statistically significant. However, the expression of miR-92a remarkably declined in the LNA-anti-miR group compared with the control groups (either Scrambled LNA or untreated group) (4.8 \pm 2.06 vs. $-1.9 \pm 2.2$, respectively; $\mathrm{P}=0.04$ ) (Figure 1). Despite the decreased expression of miR181a after LNA transfection compared to the untreated group, the difference was not statistically significant $(7.2 \pm 2.7$ vs. $5.4 \pm 2.9$, respectively; $\mathrm{p}=$ 0.6 (Figure 1).

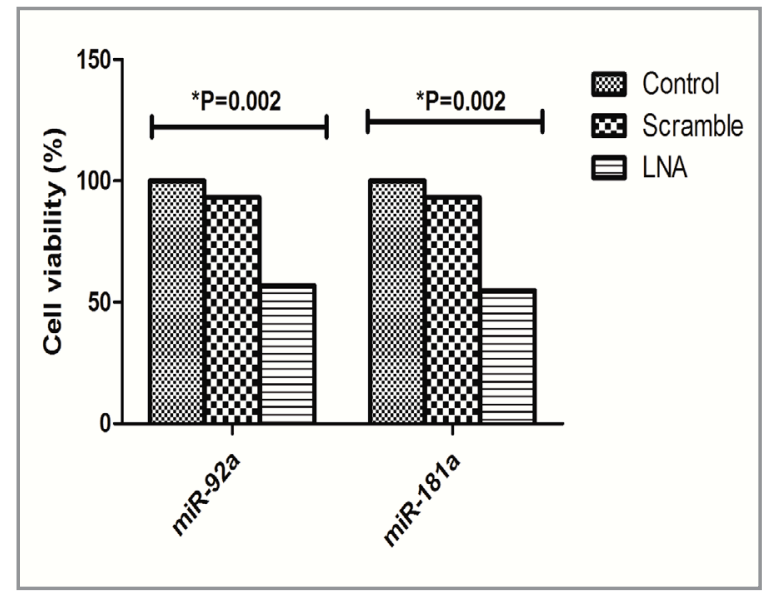

Figure 2. The effect of miR-92a and miR-181a blockage with LNA-anti-miR on the cell viability The viability of untreated cells was considered as $100 \%$ and the viability of other groups (either scrambled or LNA) was compared to untreated group cell was evaluated using MTT assay. Data are represented of the mean $\pm S D$ of five independent repeated experiments. The Pvalue is indicative of the comparison between LNA-anti-miR transfected and control group.

\section{Blockage of miR-92a and miR-181a Significant- ly Reduce the Viability of HL-60 Cell Line}

To test the effect of miR-92a and miR-181a blockage on cell viability, MTT assay was performed at $72 \mathrm{~h}$ after cell transfection. As was predicted, the viability of HL-60 cell line reduced slightly in the scrambled-LNA group compared to untreated control group, but it was greatly decreased about 50\% in LNA-anti-miR-92a and LNA-anti-miR-181a transfected group compared to both control groups after $72 \mathrm{~h}$ post transfection $(\mathrm{p}=0.002)$ (Figure 2).

\section{WT1, c-Kit and CEBPA Expression After LNA- anti-miRNA Transfection}

After successful blocking of the miR-92a and miR-181a by transfection of HL-60 cell lines with LNA-anti-miRNA, the expression of WT1, c-Kit and CEBPA genes was evaluated to show whether miR-92a and miR-181a blockage can change WT1, c-Kit, and CEBPA expression.

The results showed that when HL-60 cell lines were transfected with LNA-anti-miR92a, the mean expression of c-Kit was significantly decreased in transfected group compared to non-transfected one ( $6.2 \pm 2.7$ vs. $4.2 \pm 2.5$, respectively; $\mathrm{p}=0.01)$, where- 


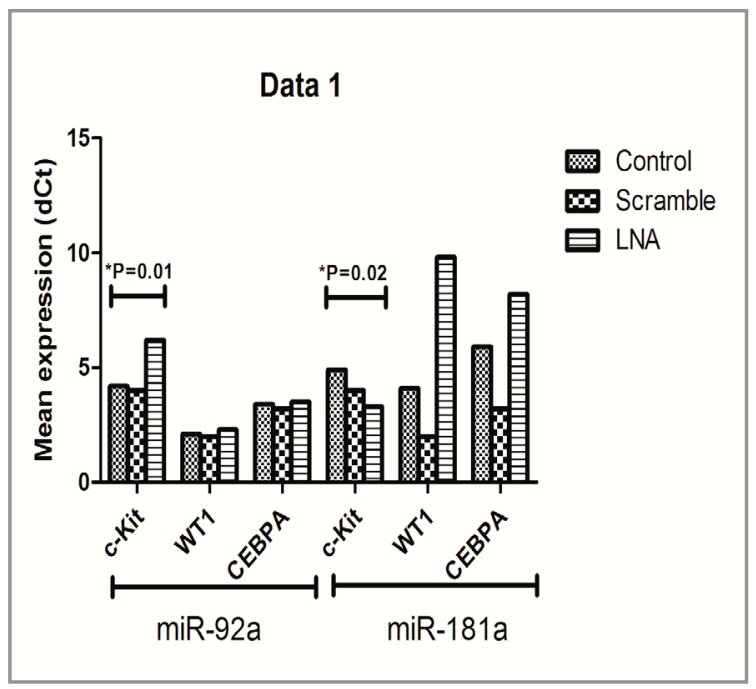

Figure 3. Change in c-Kit, WT1 and CEBPA gene expression following miR-92a and miR-181a blockage with LNA-antimiR The mean \pm SD of dCt for c-Kit, WT1 and CEBPA were compared between LNA group and control groups (either scrambles or untreated). Data are represented of the five independent repeated experiments. The P-value is indicative of the comparison between LNA-anti-miR transfected and control group.

as, the mean expression of WT- 1 and CEBPA did not significantly change in transfected group compared to non-transfected one $(2.3 \pm 1.3$ vs. $2.1 \pm 1.6$, respectively; $\mathrm{p}=0.2$ and $3.5 \pm 3.2$ vs. $3.4 \pm 1.9$, respectively; $\mathrm{p}=0.9$ ) (Figure 3).

Conversely, in case of cell transfection with LNAanti-miR-181a, the mean expression of c-Kit was significantly increased in transfected group compared to non-transfected one $(3.3 \pm 1.4$ vs. $4.9 \pm 2.8$, respectively; $\mathrm{p}=0.02$ ), while, the mean expression of WT1 was decreased in transfected group compared to non-transfected one which was near the significant level $(9.8 \pm 1.2$ vs. $4.1 \pm 2.3 ; \mathrm{p}=0.05)$. Also, the difference was not statistically significant for CEBPA gene $(8.2 \pm 1.2$ vs. $5.9 \pm 2.1 ; \mathrm{p}=0.3)$ (Figure 3 ).

\section{DISCUSSION}

Recently, evaluation of miRNAs signature has been used as a non-invasive tool for the diagnosis and management of AML patients with a focus on the modification of miRNAs as a new therapeutic method. In this regard, many efforts on targeting
miRNA expression in cancers either directly by use of oligonucleotides or virus-based constructs or administration of strategies involving the use of drugs which regulate miRNA expression have been performed..$^{15}$ Besides, there is an emerging role of miRNAs to alter the sensitivity of cancer patients to treatment making them a suitable target for the management of patients who are resistant to chemotherapy treatment. ${ }^{15,16}$

In the current study, we aimed to determine the expression of miR-92a and miR-181a as a new molecular biomarker in AML patients. Also, we evaluated for the first time the consequence of miR-92a and miR-181a blockage by LNA-antimiR-92a and LNA-anti-miR-181a on the expression of WT1, CEBPA and c-Kit genes which are frequently dysregulated in AML patients. Our results demonstrated that both miR-92a and miR181a are highly overexpressed in AML patients compared to healthy controls. Aberrant expression of miR-92a family and miR-181a has been reported in AML patients. Tanaka et al found that miR-92a was strongly increased in leukemic cells from both AML and ALL patients while its plasma level dramatically decreased in the plasmas of acute leukemia patients compared to healthy control group. ${ }^{17}$ Ohyashiki et al. demonstrated that the cell to plasma ratio of miR-92a expression was significantly higher in both AML and ALL cells compared with PBMC from healthy controls. ${ }^{18} \mathrm{Al}-$ though the source of miR-92a analysis was different between our study (PBMCs) compared to these studies (leukemia cells and plasma level), these data suggest that miR-92a may be implicated as an oncomiR in both plasma and leukemia cells as well as PBMCs of AML patients and so, could be a novel biomarker for monitoring these patients. Furthermore, as mentioned by Tanaka et al, miRNAs are assembled inside exosome which then can be uptake by cancer cells. ${ }^{17}$ Whether the PBMCs have the same capacity as cancer cells to take exosomebearing miRNAs needs to be clarified. However, assessment of the serum miR-92a and miR-181a level and correlation between PBMCs to the serum level of these miRNAs may provide useful data to understand the clinical significance of miR-92a and miR-181a in the PBMCs of our AML patients. 
The second part of our results showed that blockage of the miR-92a and miR-181a in HL-60 cell line remarkably reduces cell viability.

There are various reports describing that miR-92a and miR-181a are involved in cell cycle regulation and cell signaling by targeting genes promoting cell differentiation, apoptosis and those inhibiting cell proliferation. ${ }^{19-21}$ In two separate study by Sharifi et al., inhibition of miR-92a with LNA-anti-miR92a, reduced survival and induced cell apoptosis and necrosis in human acute megakaryoblastic (M07e) and acute promyelocytic leukemia (HL-60) cell line. ${ }^{22,23}$ Liu et al. explained that transfection of HL-60 cell line with miR-181a mimic significantly increased G1/S cell cycle transition and cell proliferation suggesting that miR-181a may contribute to AML progression by promoting myeloid cell proliferation. $^{24}$

In addition, our study revealed that inhibition of miR-92a with LNA-anti-miR-92a significantly decreased c-Kit level. Conversely, the c-Kit expression was upregulated following miR-181a blockage. No significant change was observed about WT-1 and CEBPA expression following either miR-92a or miR-181a blockage.

It has been demonstrated that activation of the receptor tyrosine kinases (RTKs), c-Kit and its intracellular signal transduction pathway including KRAS, NRAS and MAPK1 have a critical role in regulation of the cellular function including cell proliferation, survival as well as apoptosis highlighting that targeting these molecules could be a known as a novel therapeutic approaches for treatment of cancers especially hematologic malignancies. ${ }^{25}$ In hematopoietic system, the c-Kit receptor (CD117) and its ligand, stem cell factor (SCF) are considered to play a critical role in hematopoiesis by promoting growth and differentiation of early normal hematopoietic progenitors and leukemic cells in bone marrow. ${ }^{26}$ Approximately $80 \%$ of the AML cases express the proto-oncogene c-Kit and its expression is used as a reliable molecular marker indicating poor prognosis of AML patients. ${ }^{26,27}$ We also observed in this study that the expression of c-Kit was significantly increased (11.5 fold) in our AML patients compared to group control $(\mathrm{p}=$ 0.01). Study by Huang et al. revealed that by bind- ing to 3 -untranslated regions (UTRs), miR-181a can regulate cell proliferation via downregulation of the KRAS, NRAS and MAPK1 signaling molecules, thus decreases AML cell growth. ${ }^{28}$ Consistent with these results, Nanbakhsh et al. also showed that overexpression of miR-181a in AML blasts is associated with attenuation of their resistance to daunorubicin (DNR) and NK-cell-mediated killing, supporting that high miR-181a might be implicated in the chemosensitization of leukemic cells to DNR and NK cells-mediated lysis. ${ }^{21}$

Unexpectedly, we observed that miR-181a but not miR-92a is upregualted in high risk AML patients. However, in a meta-analysis by Guo et al., it was showed that elevated miR-181 expression was associated with increased survival in American AML patients, while with reduced survival in Chinese patients, indicating that miR-181 can be used as a prognostic biomarker in AML patients depending on the origin of patient population. ${ }^{29}$ A possible explanation for this part of our results is that the risk stratification of our AML patients was based on cytogenetic status and FLT3 mutation since we have no data about other gene mutation status including NPM1 and CEBPA and also FLT3 allelic ratio. ${ }^{14}$ Therefore, considering other gene mutation like NPM1 and CEBPA is necessary in addition to FLT-3 for exact classification of our AML patients, thereby better defining the prognostic significance of miR-181a as well as miR-92a in clinical outcome of our AML patients.

According to our results, it is possible that in addition to the downstream signaling molecules, KRAS, NRAS and MAPK1, c-Kit gene might be a new target gene for miR-92a and miR-181a which is positively and negatively regulated by these miRNAs, respectively. Regarding these findings, targeting c-Kit by LNA-anti-miR-92a and/or mimics of the miR-181a could be proposed as a novel kind of receptor tyrosin kinase inhibitors (RTKIs) for targeted therapy of AML patients along with conventional chemotherapy. More complete studies using mimics of miR-92a and miR-181a are needed to clarify whether c-Kit gene is affected directly or indirectly by miR-92a and miR-181a. 


\section{Conclusion}

We have shown that miR-92a and miR-181a are upregulated in newly diagnosed AML patients. We also demonstrated for the first time that that c-Kit gene may be a novel target gene for miR-92a and miR-181a. Regarding the anti-proliferative effect of LNA-anti-miR-92a and LNA-anti-miR-181a, regulation of miR-92a and miR-181a expression could be considered as a useful approach in line with conventional chemotherapy to limit blast cell survival and reduce leukemic cell proliferation in AML patients.

\section{REFERENCES}

1. Gabra MM, Salmena L. microRNAs and acute myeloid leukemia chemoresistance: A mechanistic overview. Front Oncol 7: 255, 2017.

2. Hanahan D, Weinberg RA. Hallmarks of cancer: the next generation. Cell 144: 646-674, 2011.

3. Rupaimoole R, Slack FJ. MicroRNA therapeutics: towards a new era for the management of cancer and other diseases. Nat Rev Drug Discov 16: 203-222, 2017.

4. Baumann V, Winkler J. miRNA-based therapies: strategies and delivery platforms for oligonucleotide and non-oligonucleotide agents. Future Med Chem 6: 1967-1984, 2014.

5. Weng H, Lal K, Yang FF, Chen J. The pathological role and prognostic impact of miR-181 in acute myeloid leukemia. Cancer Genet 208: 225-229, 2015.

6. Su R, Lin HS, Zhang $X H$, et al. MiR-181 family: regulators of myeloid differentiation and acute myeloid leukemia as well as potential therapeutic targets. Oncogene 34: 3226-3239, 2015.

7. Chen C-Z, Li L, Lodish HF, Bartel DP. MicroRNAs modulate hematopoietic lineage differentiation. Science 303: 83-86, 2004.

8. Garzon R, Croce CM. MicroRNAs in normal and malignant hematopoiesis. Curr Opin Hematol 15: 352-358, 2008.

9. Liao Q, Wang B, Li X, Jiang G. miRNAs in acute myeloid leukemia. Oncotarget 8: 3666-3682, 2017.

10. Li M, Guan X, Sun Y, et al. miR-92a family and their target genes in tumorigenesis and metastasis. Exp Cell Res 323: 1-6, 2014.

11. Mogilyansky E, Rigoutsos I. The miR-17/92 cluster: a comprehensive update on its genomics, genetics, functions and increasingly important and numerous roles in health and disease. Cell Death Differ 20: 1603-1614, 2013.
12. Elhamamsy AR, El Sharkawy MS, Zanaty AF, et al. Circulating miR-92a, miR-143 and miR-342 in Plasma are Novel Potential Biomarkers for Acute Myeloid Leukemia. Int J Mol Cell Med 6: 77-86, 2017.

13. Trino S, Lamorte D, Caivano A, et al. MicroRNAs as New Biomarkers for Diagnosis and Prognosis, and as Potential Therapeutic Targets in Acute Myeloid Leukemia. Int J Mol Sci 19: 460, 2018.

14. Döhner H, Estey E, Grimwade D, et al. Diagnosis and management of AML in adults: 2017 ELN recommendations from an international expert panel. Blood 129: 424-447, 2017.

15. Iorio MV, Croce CM. MicroRNA dysregulation in cancer: diagnostics, monitoring and therapeutics. A comprehensive review. EMBO Mol Med 4: 143-159, 2012.

16. Magee P, Shi L, Garofalo M. Role of microRNAs in chemoresistance. Ann Transl Med 3: 332, 2015.

17. Tanaka M, Oikawa K, Takanashi M, Kudo M, Ohyashiki J, Ohyashiki K, et al. Down-regulation of miR-92 in human plasma is a novel marker for acute leukemia patients. PLoS One 4: e5532, 2009

18. Ohyashiki JH, Umezu T, Kobayashi C, et al. Impact on cell to plasma ratio of miR-92a in patients with acute leukemia: in vivo assessment of cell to plasma ratio of miR-92a. BMC research notes 3: 347, 2010.

19. Mi S, Li Z, Chen P, et al. Aberrant overexpression and function of the miR-17-92 cluster in MLL-rearranged acute leukemia. Proceedings of the National Academy of Sciences 107: 3710-3715, 2010.

20. Rao E, Jiang C, Ji M, Huang X, lqbal J, Lenz G, et al. The miRNA-17 92 cluster mediates chemoresistance and enhances tumor growth in mantle cell lymphoma via PI3K/AKT pathway activation. Leukemia 26: 1064-1072, 2012.

21. Nanbakhsh A, Visentin G, Olive D, et al. miR-181a modulates acute myeloid leukemia susceptibility to natural killer cells. Oncoimmunology 4: e996475, 2015.

22. Sharifi M, Salehi R. Blockage of miR-92a-3p with locked nucleic acid induces apoptosis and prevents cell proliferation in human acute megakaryoblastic leukemia. Cancer Gene Ther 23: 29-35, 2016.

23. Sharifi M, Salehi R, Gheisari $Y$, Kazemi M. Inhibition of MicroRNA miR-92a inhibits cell proliferation in human acute promyelocytic leukemia. Turk J Haematol 30: 157-162, 2013.

24. Liu X, Liao W, Peng H, et al. miR-181a promotes G1/S transition and cell proliferation in pediatric acute myeloid leukemia by targeting ATM. J Cancer Res Clin Oncol 142: 77-87, 2016.

25. Stankov K, Popovic S, Mikov M. C-KIT signaling in cancer treatment. Curr Pharm Des 20: 2849-2880, 2014.

26. Ikeda H, Kanakura $\mathrm{Y}$, Tamaki $\mathrm{T}$, et al. Expression and functional role of the proto-oncogene c-kit in acute myeloblastic leukemia cells. Blood 78: 2962-2968, 1991. 
International Journal of Hematology and Oncology

27. Gao X, Lin J, Gao L, et al. High expression of c-kit mRNA predicts unfavorable outcome in adult patients with $t(8 ; 21)$ acute myeloid leukemia. PLoS One 10: e0124241, 2015.

28. Huang $X$, Schwind S, Santhanam R, et al. Targeting the RAS/ MAPK pathway with miR-181a in acute myeloid leukemia. Oncotarget 7: 59273-59286, 2016.

29. Guo Q, Luan J, Li N, et al. MicroRNA-181 as a prognostic biomarker for survival in acute myeloid leukemia: a metaanalysis. Oncotarget 8: 89130-89141, 2017.

\section{Correspondence:}

Dr. Mani RAMZI

Hematology Research Center and Department

of Bone Marrow Transplantation

Shiraz University of Medical Sciences,

SHIRAZ / IRAN

Tel: 009836122263

e-mail: ramzim96@outlook.com 